Article

\title{
Application of Biodegradable PLGA-PEG-PLGA/CPC Composite Bone Cement in the Treatment of Osteoporosis
}

\author{
Chao Guo ${ }^{\dagger}$, Dongyang Niu ${ }^{\dagger}$, Jia Liu, Xiaogang Bao and Guohua Xu * \\ Department of Orthopedic Surgery, Spine Center, Second Affiliated Hospital of Naval Military Medical \\ University, Shanghai 200003, China; 18640813178@163.com (C.G.); a1003822552@163.com (D.N.); \\ 13506191593@163.com (J.L.); bxg1832178@smmu.edu.cn (X.B.) \\ * Correspondence: xuguohuamail@smmu.edu.cn; Tel.: +86-13386279098; Fax: +86-21-81885647 \\ + These authors contributed equally to this work.
}

check for

updates

Citation: Guo, C.; Niu, D.; Liu, J.; Bao, X.; Xu, G. Application of Biodegradable PLGA-PEG-PLGA/ CPC Composite Bone Cement in the Treatment of Osteoporosis. Coatings 2021, 11, 827. https://doi.org/ 10.3390/coatings11070827

Received: 25 May 2021

Accepted: 6 July 2021

Published: 9 July 2021

Publisher's Note: MDPI stays neutral with regard to jurisdictional claims in published maps and institutional affiliations.

Copyright: (c) 2021 by the authors. Licensee MDPI, Basel, Switzerland. This article is an open access article distributed under the terms and conditions of the Creative Commons Attribution (CC BY) license (https:// creativecommons.org/licenses/by/ $4.0 /)$.
Abstract: The aim of this study was to evaluate the biological activity, safety, and effectiveness of poly(lactic acid)-poly(glycolic acid)-poly(ethylene glycol)-calcium phosphate cement (PLGAPEG-PLGA/CPC). Methods: The PLGA-PEG-PLGA/CPC composite bone cement was used for interaction with MC3T3-E1 mouse osteoblasts in vitro and its compatibility was tested using Cell Counting Kit-8 (CCK-8). Alizarin Red staining and alkaline phosphatase activity were used to detect the osteogenic properties. Twenty healthy female New Zealand rabbits were selected to establish osteoporosis models, which were randomly divided into two groups. The experimental group was treated with $30 \mathrm{wt}$.\% PLGA-PEG-PLGA/CPC, while the control group was treated with polymethyl methacrylate (PMMA) bone cement. Imaging and histomorphology of the vertebral body were analyzed after 12 weeks. The distribution and degradation of bone cement were assessed using micro-computed tomography examination and hematoxylin-eosin (HE) staining. Results: In vitro, CCK-8 revealed significant proliferation of osteoblasts in the PLGA-PEG-PLGA/CPC composite bone cement. Alizarin Red staining showed that the degree of staining increased with time. Quantitative results showed that absorbance was significantly higher in this group than in the CPC group on days 7 and 14. The alkaline phosphatase activity levels on days 7 and 14 were significantly higher in the $30 \mathrm{wt}$.\% PLGA-PEG-PLGA/CPC group than in the CPC group. In vivo, postoperative microcomputed tomography and histomorphology showed that the material was evenly distributed in the vertebral body and a small amount penetrated into the trabecular bone. After 12 weeks, CPC degradation and absorption and the formation of new bone matrix were observed and the formation of a callus was obvious. Conclusion: PLGA-PEG-PLGA/CPC composite bone cement has a positive effect on the treatment of osteoporosis.

Keywords: osteoporosis; bone cement; biodegradation

\section{Introduction}

Severe osteoporotic lower back pain and osteoporosis-related vertebral fractures (OVCF) have become global diseases that affect the lives and health of elderly people. Percutaneous vertebroplasty, including percutaneous vertebroplasty (PVP) and percutaneous balloon dilatation (PKP), is currently one of the main treatments for elderly osteoporotic lower back pain and OVCF [1,2], which produces a good short-term therapeutic effect.

Regardless of the PVP or PKP technology, filling materials are some of the core parts of the treatment. At present, bone cements used for vertebral body strengthening are mainly made of polymethyl methacrylate (PMMA), and there is no special bone cement for osteoporotic spine uses in clinical applications [3]. The development of a special bone cement for vertebral body shaping has become a research focus [4].

In addition to polymer medical materials used for filling materials, biomedical inorganic materials have also received increasing attention in recent years [3]. Among these materials, biodegradable and biologically active inorganic bone cement materials have 
gradually attracted attention [4,5]. Calcium phosphate cement (CPC) is a self-setting material. CPC has broad application prospects in PVP filling materials; however, the clinical application of CPC in the weight-bearing bones of the spine has many drawbacks, such as its low compressive strength, poor anti-collapse properties, and poor injection performance. As such, the current application of CPC in spinal repair requires further research [6-8].

Based on the aforementioned problems, our laboratory synthesized a new type of biodegradable composite bone cement system, namely poly(lactic acid)-poly(glycolic acid)-poly(ethylene glycol)-calcium phosphate cement (PLGA-PEG-PLGA/CPC) [9]. The material was compounded with $\alpha$-tricalcium phosphate $(\alpha-\mathrm{TCP}), \mathrm{CaCO} 3$, and $\mathrm{CaHPO} 4$ to form a composite CPC system ( $\alpha$-TCP-MCPM-CC system), focusing on solving the problems related to the inorganic bone cement setting time, compressive strength, and acid-base balance. Furthermore, the PLGA-PEG-PLGA triblock polymer temperaturesensitive hydrogels, which have been used in clinical applications, allow liquid-solid-phase transitions and high temperatures under different temperature conditions. Molecular polymers can improve the adhesiveness of bone cement and are mixed with $\mathrm{NaH}_{2} \mathrm{PO}_{4}$ in proportion to replace the traditional inorganic liquid phase of a bone cement. Quickly mixing the composite CPC system to form a PLGA-PEG-PLGA/CPC bone cement system solves the problem of CPC use in the injection process. It is easy to produce the filter pressure effect and cause the solid-liquid separation phenomenon. The filling material is easily diluted by blood in the cancellous bone and has advantages of strong compressive strength, high ductility, good injection performance, and strong water resistance. Finally, this system is transformed into the hydroxyapatite component of biological bone. This makes it more in line with the requirements for spinal repair and PVP operation and provides a theoretical and experimental basis for the development of special bone cements for spinal repair. This study was aimed at detecting the in vitro biological activity of the bone cement of the system in animal experimental studies and evaluating its biological activity, safety, and effectiveness in vivo and in vitro.

\section{Materials and Methods}

\subsection{In Vitro Cell Compatibility Evaluation}

MC3T3-E1 mouse osteoblasts (Bohu Biotechnology Co., Ltd., Shanghai, China) were cultured and heated on a CPC bone cement column and PLGA-PEG-PLGA/CPC composite bone cement (10 wt.\%, $20 \mathrm{wt} . \%, 30 \mathrm{wt} . \%$, and $40 \mathrm{wt} . \%$ PLGA-PEG-PLGA/CPC) column at $130{ }^{\circ} \mathrm{C}$ for $10 \mathrm{~min}$. The cells were cooled to $25^{\circ} \mathrm{C}$, soaked in $75 \%$ ethanol for $2 \mathrm{~h}$, and then soaked in MEM- $\alpha$ medium (containing 10\% fetal bovine serum) (Gibco, Grand Island, NY, USA) for $4 \mathrm{~h}$. The cells were re-cultured in 24-well plates and analyzed using a CCK-8 kit (Dojindo, Kumamoto, Japan) $24 \mathrm{~h}$ later. The relative growth rate (RGR) of the cells was calculated based on the optical density (OD) value, using the following formula: RGR = (OD value of the experimental group/OD value of the blank control group) $\times 100 \%$. When RGR $>75 \%$, the cell compatibility of the material was considered to be good; when RGR > $100 \%$, the material was considered to promote cell proliferation.

\subsection{Alizarin Red Staining and Quantitative Detection}

Two parallel samples were taken for each ratio of the PLGA-PEG-PLGA/CPC composite bone cement column (as mentioned above) and cut into powder. The mixed culture liquid was sterilized at a concentration of $10 \mathrm{mg} / \mathrm{mL}$. The stem cells were seeded in 24-well plates. After $24 \mathrm{~h}$, the old culture medium was removed and $0.5 \mathrm{~mL}$ of osteogenic induction medium (Sigma, Hiroshima, Japan) was added. Induction was performed for 7-14 days. The cells were then fixed for $15 \mathrm{~min}$ with $4 \%$ paraformaldehyde (Sigma, Japan), stained with Alizarin Red staining solution (Beyotime, Petaluma, CA, USA) for 3 min, observed under a microscope, and photographed. Subsequently, hexadecyl chloride was added and left at room temperature for $30 \mathrm{~min}$, the supernatant was drawn, then the absorbance of the sample was measured at $560 \mathrm{~nm}$ using a microplate reader (Biotech, Shanghai, China), while the absorbance of the supernatant of a group of simple cells was measured at the 
same time. The measured value of the sample was the difference between the absorbance values of the sample and the simple cell group.

\subsection{Quantitative Detection of Alkaline Phosphatase (ALP) Activity}

Grouping and culturing were performed as described above. The cells were lysed (Beyotime, USA), the supernatant was taken, and the alkaline phosphatase kit (Beyotime, USA) was used to test samples. The absorbance of each sample was examined three times and the average value was recorded.

\subsection{Rabbit Model of Osteoporosis}

Osteoporosis was induced using the classic method of ovariectomy, as has been previously reported in the literature [10,11]. In 20 New Zealand rabbits, a $3 \mathrm{~cm}$ midline incision was made in the abdomen (approximately $3 \sim 4 \mathrm{~cm}$ from the upper edge of the lowest nipple as the midpoint) into the abdominal cavity, then the fallopian tubes were ligated and the ovaries were removed.

\subsection{Rabbit Vertebroplasty Model}

The 20 experimental rabbits used in the preosteoporosis model were divided into two groups, A and B (10 rabbits in each group, weighing 2.5 3.0 kg). Group A was the experimental group (PLGA-PEG-PLGA/CPC composite bone cement), while group B was the control group (PMMA bone cement). Using the bilateral iliac bones corresponding to the upper edge of the lumbar 7 vertebral body as a mark, the spinous processes and kyphotic processes of the L5 and L6 vertebral bodies were located. Centering on the upper articular processes of L5 and L6, a 4-6-cm straight longitudinal incision approximately along the line of the two articular processes was made, exposing the upper articular process and mastoid process of the spine, while the transverse processes were also explored. We continued to separate the base of the vertebral body on the cranial side, exposing the vertebral accessory process until we reached the tail in the intervertebral foramen. The intersection of the vertical line of the midpoint of the spine mastoid and the horizontal line of the lower edge of the spine was used as the puncture point. A manual electric drill was used to gradually break through the cortex and then to gradually penetrate into the vertebral body. The four walls were probed to ensure that the four walls of the needle track were intact without breaking through the surrounding bone cortex. Subsequently, the 2 3 $\mathrm{mm}$ bone tissue puncture needle was used to expand the channel. After probing the integrity of the four walls, bone cement injection was performed. Twelve weeks after the operation, the rabbits in each group were sacrificed and the experimental vertebral body segments were removed for imaging and histomorphological analysis.

\subsection{Statistics}

Statistical Product and Service Solutions (SPSS) software (IBM version 21) was used for data processing. Normally distributed quantitative data are expressed as $\pm \mathrm{s}$, while the related data for the D1 D5 continuous normal distribution were compared with one-way analysis of variance (Duncan method) to analyze the significance of differences between multiple groups of samples, whereby $p<0.05$ indicated that differences were statistically significant.

\section{Results}

3.1. PLGA-PEG-PLGA/CPC Composite Bone Cement Significantly Promoted the Proliferation of Osteoblasts and Had Osteoinductive Properties

Cell proliferation activity was detected using CCK- 8 colorimetry. The results showed that the cell proliferation ability of the PLGA-PEG-PLGA/CPC composite bone cement group increased with increasing PLGA-PEG-PLGA content, while the cell proliferation activity reached a maximum on the third day with the increase in PLGA-PEG-PLGA content (Figure 1A). To further compare the cell proliferation of each group, the concept of 
the "relative growth rate" was introduced. In the statistical analysis, the relative growth rates of cells in each proportion of composite bone cement were above $90 \%$ (Figure 1B). The cell growth rate of $40 \mathrm{wt} . \%$ PLGA-PEG-PLGA/CPC on the third day was comparable to that of $\mathrm{CPC}$ bone cement alone. The relative growth rate of cement was significantly different $(p=0.032)$.

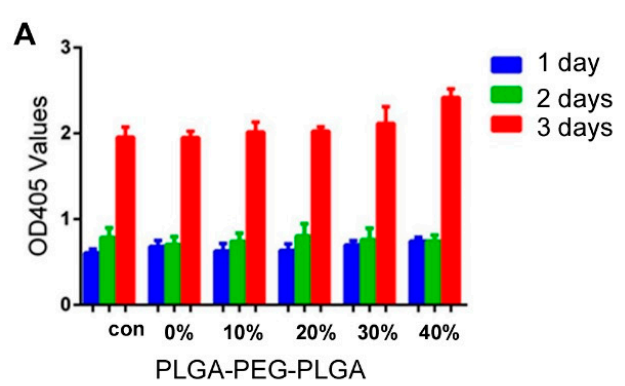

C
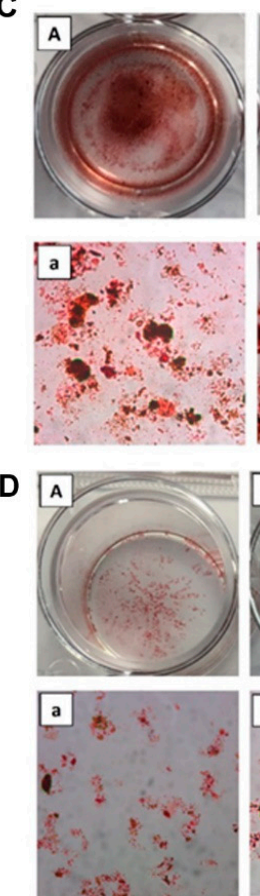

E
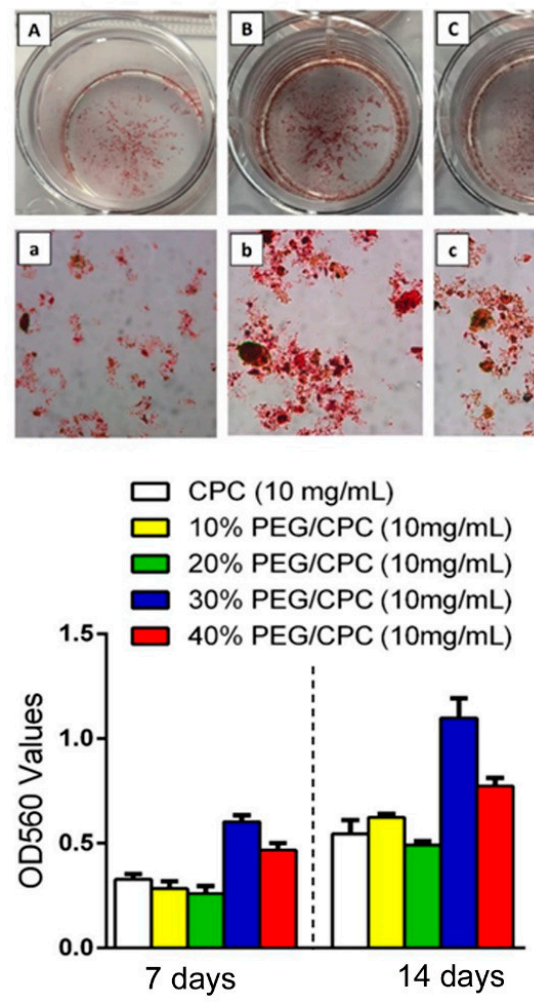
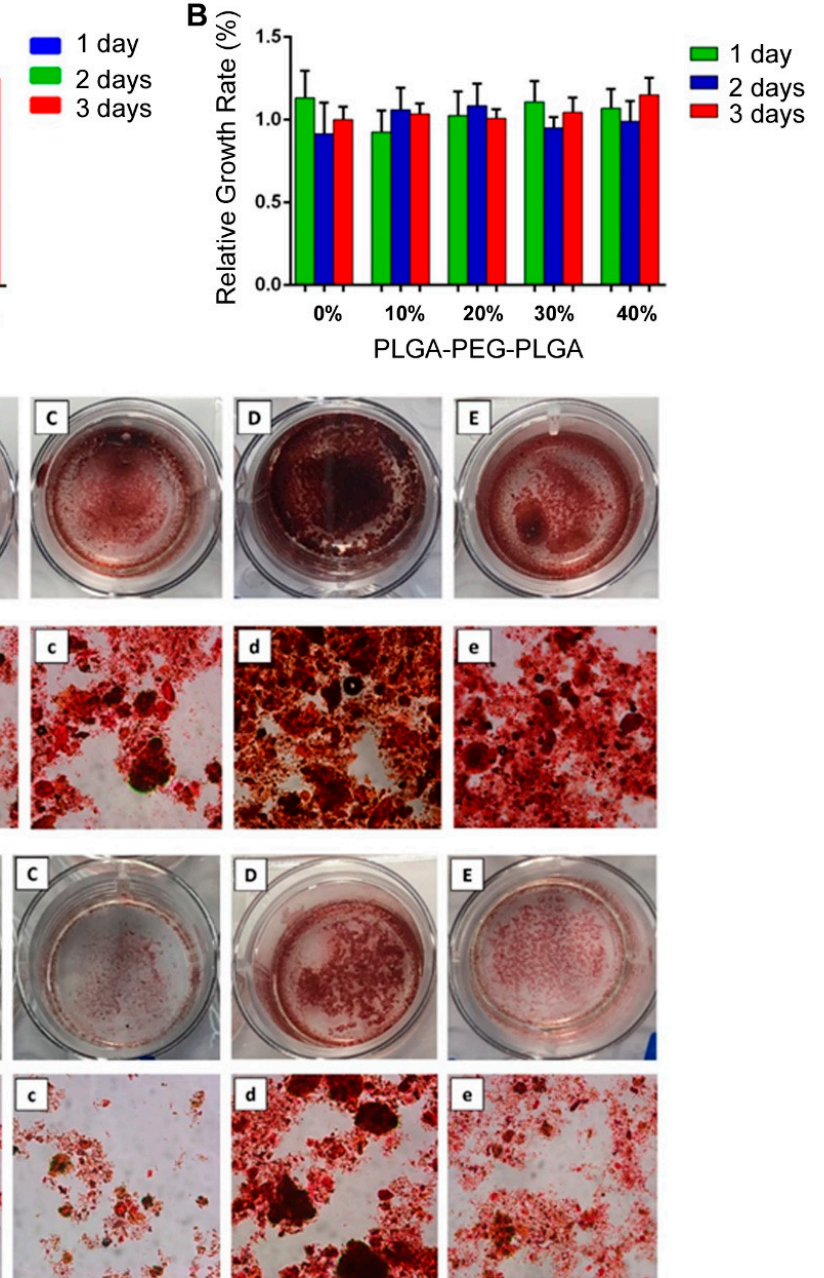

$F$

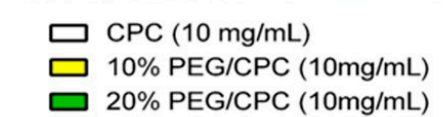

$\square 20 \%$ PEG/CPC (10mg/mL)

$30 \%$ PEG/CPC $(10 \mathrm{mg} / \mathrm{mL})$

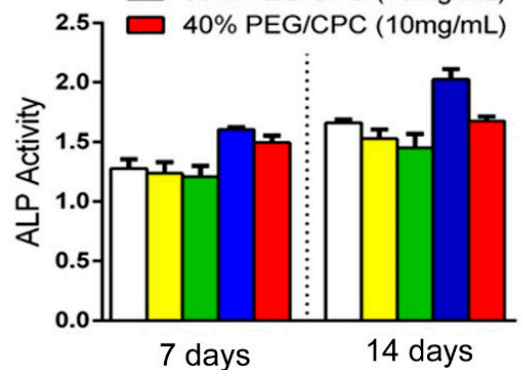

7 days
14 days

Figure 1. PLGA-PEG-PLGA/CPC composite bone cement significantly promotes the proliferation of osteoblasts and osteoinductive properties: (A) cytocompatibility of PLGA-PEG-PLGA/CPC bone 
cement; (B) relative growth rate of each proportion at different times (40 wt.\% vs 0 wt. $\%: p=0.032$ ), with Alizarin Red stain results for various proportions in culture for 7 days (C) and 14 days (D) (A-E are $0 \mathrm{wt} . \%, 10 \mathrm{wt} \%$, $20 \mathrm{wt} . \%, 30 \mathrm{wt} . \%$, and $40 \mathrm{wt} . \%$ PLGA-PEG-PLGA/CPC, respectively; a-e are the microscope images of A-E, respectively); (E) quantitative results of Alizarin Red staining in each group (30 wt. $\%$ and 40 wt. $\%$ vs. 0 wt. $\%: p=0.028$ ); (F) quantitative ALP activity results (30 wt. $\%$ vs 0 wt. $\%: p=0.035$ ) (OD405: optical density 405, OD560: optical density 560).

Alizarin Red staining showed that the staining degree of each group increased gradually with time (Figure 1C,D). After 7 days, the staining degrees of Alizarin Red in the $10 \mathrm{wt} . \%$ and $20 \mathrm{wt} . \%$ PLGA-PEG-PLGA/CPC groups were not significantly different from that of CPC bone cement alone, but both groups showed increases in the level of staining over time. The formation of calcified nodules in the $10 \mathrm{wt} . \%$ and $20 \mathrm{wt} . \%$ PLGA-PEGPLGA/CPC groups was observed, showing a darker red color on day 14. The degrees of staining in the $30 \mathrm{wt} . \%$ and $40 \mathrm{wt} . \%$ PLGA-PEG-PLGA/CPC groups were significantly higher on the 7th day than that in the CPC bone cement alone group. After 14 days, the $30 \mathrm{wt} . \%$ group was macroscopically observed to show a significant difference, suggesting that Alizarin Red and cytoplasmic calcium ions combine to form a large number of red calcium nodules. The quantitative results (Figure 1E) showed that the absorbance in each group gradually increased over time. After 7 and 14 days, the PLGA-PEG-PLGA/CPC in each group was higher than that in the CPC group, with the differences in the $30 \mathrm{wt} . \%$ and $40 \mathrm{wt} . \%$ PLGA-PEG-PLGA/CPC groups reaching statistical significance ( $p=0.028$; therefore, as the content of PLGA-PEG-PLGA increased, the absorbance value of Alizarin Red staining gradually increased and then gradually decreased. The activity in the $30 \mathrm{wt} . \%$ PLGA-PEG-PLGA/CPC group was the highest.

Over time, the change in ALP activity in each group of materials (Figure 1F) showed that after 7 and 14 days, ALP activity levels in the $10 \mathrm{wt} . \%$ and $20 \mathrm{wt} . \%$ PLGA-PEG-PLGA groups were lower than in the CPC group. When the PLGA-PEG-PLGA content increased to $30 \%$, the ALP activity levels on the 7th day $(1.61 \pm 0.08)$ and 14 th day $(2.03 \pm 0.12)$ were significantly higher than in the simple CPC group $(1.28 \pm 0.10$ and $1.66 \pm 0.03$, respectively; $p=0.035$ ), while the $30 \mathrm{wt} . \%$ PLGA-PEG-PLGA/CPC group demonstrated the highest activity. This indicates that the PLGA-PEG-PLGA/CPC composite bone cement significantly promotes osteoblast proliferation and osteoinductive properties.

\subsection{Postoperative Imaging of PLGA-PEG-PLGA/CPC Composite Bone Cement in the Treatment of Osteoporosis with PVP in Rabbits}

Twelve weeks after PVP, micro-CT of rabbit experimental specimens in group A showed that the bone cement was partially absorbed and was tightly combined with the new bone trabecula. There were dense trabecular bones in the vertebral body around the bone cement and the bone cement vertebral body was implanted. A large number of calluses were formed nearby (Figure 2A). The experimental specimens of the rabbits in group $\mathrm{B}$ showed that the bone cement was not absorbed and was fully wrapped by the surrounding bone trabeculae. Bone hyperplasia was observed near the vertebral bodies. There was no obvious new bone formation on the side of the vertebral body implantation. The bone cement density was quite different from the surrounding bone tissue density (Figure 2B). 

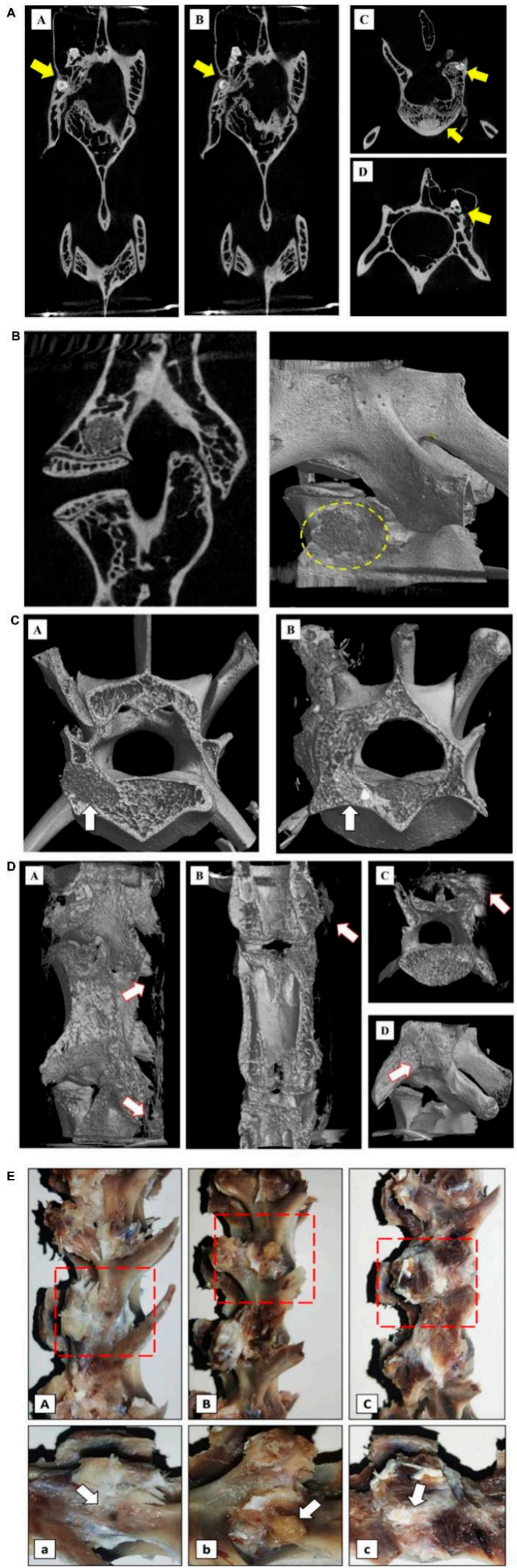

Figure 2. Postoperative imaging examination of PLGA-PEG-PLGA/CPC composite bone cement in the treatment of osteoporosis in rabbit vertebroplasty: (A) results of the vertebral body and micro-CT 
of the PLGA-PEG-PLGA/CPC group 12 weeks after surgery, showing the distribution and location of the implanted material in the vertebral body (A/B coronal plane; C/D level), where the yellow arrows indicate implant material and C/D shows the surrounding callus formation; (B) results of the vertebral body and micro-CT of PMMA implantation material 12 weeks after surgery, showing the distribution and location of the implantation material in the vertebral body (exposed PMMA can be seen around the vertebral body, without obvious callus formation) (A: sagittal plane; B: three-dimensional reconstruction); (C) comparison of perivertebral osteogenesis between the PMMA group and the PLGA-PEG-PLGA/CPC group at 12 weeks postoperatively indicates the arrow of bone cement implantation (A is the PMMA group and the density difference between the implant material and the surrounding was great; $B$ is the PLGA-PEG-PLGA/CPC group and the density difference between the implant material and the cancellous bone was small; the osteogenesis in and out of the vertebral body was obvious and the surrounding bone callus was formed); (D) comparison of bone callus around vertebral body 12 weeks after PMMA bone cement was made between PLGAPEG-PLGA/CPC group A and group B (A-C: group A, PLGA-PEG-PLGA/CPC bone cement group, obvious vertebral osteogenesis and irregular callus formation around the vertebral body; D: group B, PMMA bone cement group, no obvious osteogenesis around the vertebral body, no obvious callus formation); (E) the lateral profiles of the vertebrae of the blank control group, PMMA group, and PLGA-PEG-PLGA/CPC group were compared 12 weeks after surgery (A: blank control group, B: PMMA group, C: PLGA-PEG-PLGA/CPC group; Figure A, B, and C: enlarged view of the red area in Figure A, B, and C; the puncture hole indicated by arrow A has healed, without obvious callus formation around it; $\mathrm{B}$ : the arrow indicates exposed PMMA bone cement with no apparent osteogenesis around it; arrow $\mathrm{C}$ indicates the surrounding callus, where osteogenesis is evident around the puncture site).

Comparing the three-dimensional reconstructed images of the vertebral body after 12 weeks between the two experimental groups, it can be seen that the bone composition of group A was more obvious than that of group B. The proliferation of bone tissue inside the vertebral body was obvious, while the irregular callus formation on the side of the vertebral body was more obvious (Figure 2C,D). The general picture shows (Figure 2E) that in the PMMA group, exposed bone cement persisted and there was no obvious bone formation around it, while in the PLGA-PEG-PLGA/CPC group, there was no bone cement around the puncture point or bone formation around the vertebral body.

\subsection{PLGA-PEG-PLGA/CPC Composite Bone Cement Promotes the Formation of New Bone Trabeculae in Rabbits with Osteoporosis}

Tissue sections after 12 weeks (Figure 3A,B) showed that the PLGA-PEG-PLGA/CPC group presented obvious formation of a new bone matrix around the implanted material; the bone trabecula was reconstructed, osteoblasts were active, and bone formation was evident. Bone cement in the PMMA group showed unabsorbed implant material; the surrounding bone trabecula still showed signs of destruction, had not been reconstructed, and there was no obvious new bone matrix. 

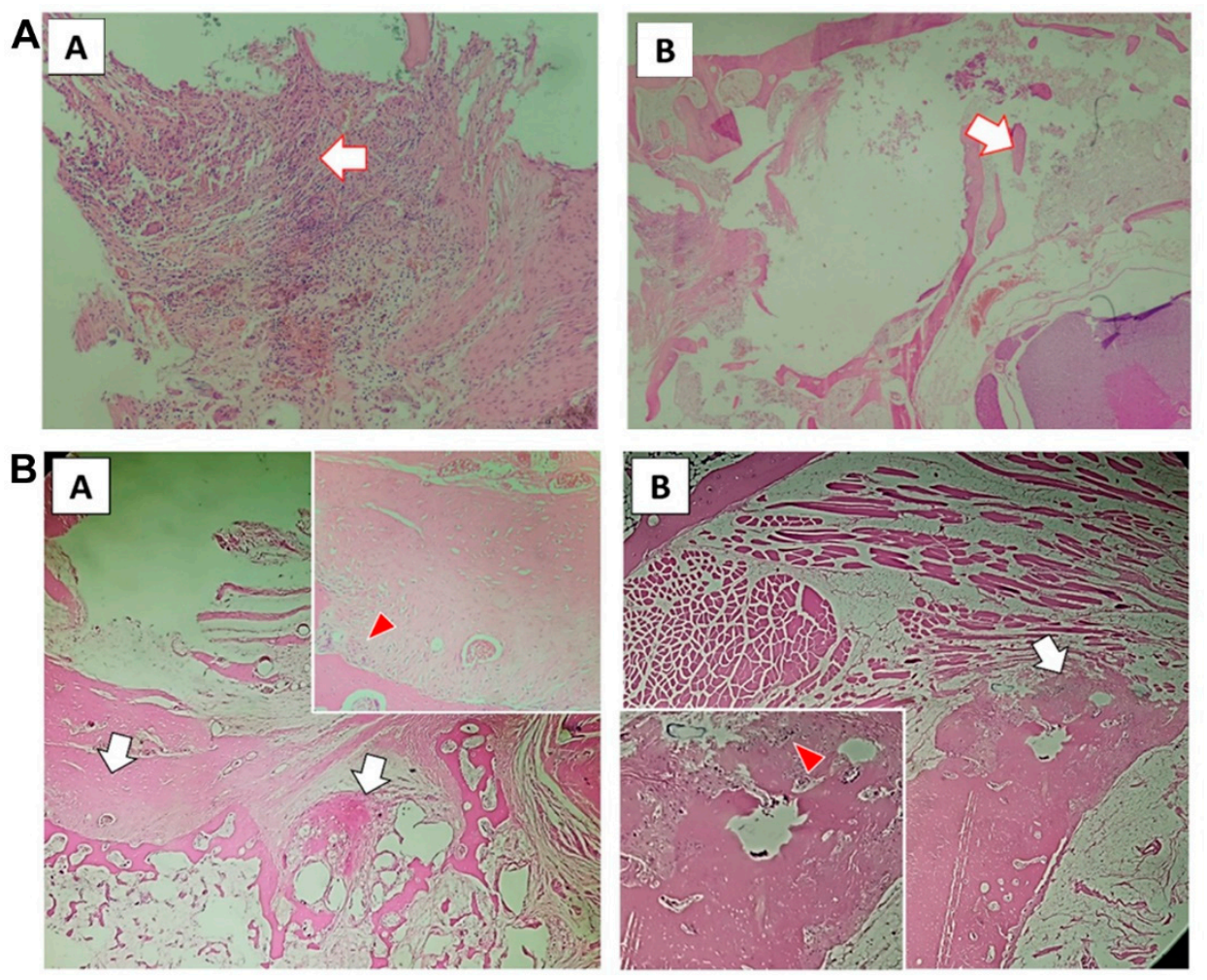

Figure 3. PLGA-PEG-PLGA/CPC composite bone cement promotes the formation of new bone trabeculae in rabbits with osteoporosis: (A) 12-week tissue section of PMMA group (A shows the macrophages surrounding the implanted material with obvious foreign body reaction; the arrow in $\mathrm{B}$ shows the damaged trabecular bone tissue, with no reconstruction or new bone matrix); (B) tissue sections of PLGA-PEG-PLGA/CPC group at 12 weeks (the white arrow in A shows the formation of new bone matrix around the original bone trabecula, with vascular tissue seen in it; the red arrow shows osteoblasts; the white arrow in picture B is the callus around the cortical bone of the vertebral body, with the new bone matrix visible inside; the red arrow is the active osteoblast).

\section{Discussion}

With the gradual research and development of self-curing CPC, the clinical application potential of injectable CPC artificial bone products has gradually attracted attention [10]. These products have certain properties, such as good biodegradability, biocompatibility [11], and bone conduction. In internal environments, they can also be mixed with blood and tissue fluid to undergo a hydration reaction and transform into the mineral phase of bone [6,12]. Among them, most of the CPC bone cement products have achieved good clinical effects and are still being improved and continuously expanded in clinical applications [13-15].

Bone cement used in the human body must have good biocompatibility. Through in vitro experiments, we proved that PLGA-PEG-PLGA/CPC composite bone cement has good cell compatibility and no toxic side effects, which may create good conditions for the proliferation of osteoblasts. The current studies suggest that biosubstitution materials cause an excessive increase in the reactive oxygen species (ROS) of osteoblasts around the material, thereby inhibiting the activity of osteoblasts, which is not conducive to the repair of the surrounding bone. The PLGA-PEG-PLGA hydrogel has physical and chemical properties similar to the human body environment, plays a role in scavenging oxygen free radicals, and promotes surrounding bone formation. Experiments have shown that the addition of PLGA-PEG-PLGA polymerization can avoid the excessive increase of intracellular ROS, reduce the degree of oxidative damage of surrounding cells to a certain extent, and increase the survival rate of cells [16]. As the temperature-sensitive hydrogel degrades, the formed pore structure is conducive to the expansion and proliferation of 
osteoblasts. Our results show that in the PLGA-PEG-PLGA/CPC composite bone cement, the addition of PLGA-PEG-PLGA polymer does not affect the osteogenic differentiation ability of MC3T3-E1, although at a certain concentration it significantly increases the material's osteogenic performance. Considering that the addition of polymer increases the surface electronegativity of the CPC material, it is more conducive to the adhesion and proliferation of MC3T3-E1 cells on the CPC material; therefore, the composite PLGAPEG-PLGA copolymer and CPC have good biological activity, with osteoblasts showing adhesion and expansion, good growth, and good osteoconductivity.

From the results of the imaging examination, it can be seen that the PLGA-PEGPLGA/CPC composite bone cement has good osteoconductivity, which is mainly related to the clear osteoinductive effect of CPC [17]. After the introduction of the temperaturesensitive hydrogel, the degradation rate of composite bone cement was faster than it was previously, which is similar to previous research results $[17,18]$. CPC materials are mainly biodegraded in two ways: one is passive absorption by chemical dissolution and the other is an active absorption process mediated by cells [13,14]; however, the degradation rate of simple CPC is relatively slow, which is not conducive to the formation of new bone in the vertebral body. Klein et al. found that CPC material used as a filler for PVP surgery gradually degrades and is absorbed over time, and that СPC material leaking out of the bone is almost completely degraded and absorbed within approximately 2 years [15]. The introduction of the PLGA-PEG-PLGA temperature-sensitive hydrogel can accelerate the degradation of the composite bone cement, while the degradation rate can be controlled by adjusting the content of the hydrogel. The experiment showed degradation of a small amount of implanted PLGA-PEG-PLGA/CPC composite bone cement at 12 weeks. This was similar to the results of previous studies. Felix et al. compounded the rapid poreforming polylactic acid-glycolic acid copolymer and CPC material. The biodegradation rate of bone cement was $55 \%$ higher than that of the CPC control group [17,18]. Over time, the vertebral body defect in the experimental group was filled with composite bone cement and there was a small amount of bone cement inside the trabecular bone structure. The $\mathrm{CPC}$ component in the bone cement has osteoinductive properties, which is beneficial to the regeneration of trabecular bone [19,20]. Through bone conduction, new bone is formed and connected to the CPC, thereby increasing the strength of the vertebral body as a whole. PMMA organic polymer materials cannot be fused with autologous bone; further, PMMA lacks biological activity and its elastic modulus does not match that of autologous bone. PMMA bone cement is associated with peripheral osteonecrosis, re-fracture of adjacent vertebrae, and material toxicity. It can be seen that the PLGA-PEG-PLGA/CPC composite bone cement has suitable biomechanical strength. Although the early strength is not as good as the PMMA material, it is conducive to the regeneration of trabecular bone in the vertebral body, while the overall vertebral body is resistant to compression over time. The compressive strength of the vertebral body continues to increase; therefore, composite bone cement has potential applications in spine bone repair.

\section{Conclusions}

In summary, we found that PLGA-PEG-PLGA/CPC composite bone cement had good biodegradability and an osteoinductive effect, which contributed to the formation of new surrounding bone trabeculae.

\section{Limitations}

This study highlights a promising bone repair material for the treatment of osteoporosis; however, at the same time, in vivo experiments should be conducted on large animals (such as pigs and sheep) to determine the clinical value of the composite material.

Author Contributions: Conceptualization, C.G. and G.X.; methodology, C.G. and X.B.; writingoriginal draft preparation, C.G. and D.N.; writing—review and editing, C.G.; visualization, D.N.; supervision, G.X.; project administration, J.L. All authors have read and agreed to the published version of the manuscript. 
Funding: This work was supported by grants from the National Natural Science Foundation of China (No. 81972076).

Institutional Review Board Statement: Not applicable.

Informed Consent Statement: Not applicable.

Data Availability Statement: The data presented in this study are available on request from the corresponding author. The data are not publicly available due to the next work.

Conflicts of Interest: The authors declare no conflict of interest.

Ethical Approval: All experiments were conducted in compliance with national guidelines and in accordance with the Guide for the Care and Use of Laboratory Animals. The study was approved by the Ethics Committee of Second Affiliated Hospital of Naval Military Medical University (NMM2019/42-1).

\section{References}

1. Clark, W.; Bird, P.; Gonski, P.; Diamond, T.H.; Smerdely, P.; McNeil, H.P.; Schlaphoff, G.; Bryant, C.; Barnes, E.; Gebski, V. Safety and efficacy of vertebroplasty for acute painful osteoporotic fractures (VAPOUR): A multicentre, randomised, double-blind, placebo-controlled trial. Lancet 2016, 388, 1408-1416. [CrossRef]

2. Bouza, C.; López-Cuadrado, T.; Almendro, N.; Amate, J.M. Safety of balloon kyphoplasty in the treatment of osteoporotic vertebral compression fractures in Europe: A meta-analysis of randomized controlled trials. Eur. Spine J. 2015, 24, 715-723. [CrossRef] [PubMed]

3. He, Z.; Zhai, Q.; Hu, M.; Cao, C.; Wang, J.; Yang, H.; Li, B. Bone cements for percutaneous vertebroplasty and balloon kyphoplasty: Current status and future developments. J. Orthop. Transl. 2015, 3, 1-11. [CrossRef] [PubMed]

4. Beig, B.; Liaqat, U.; Douna, I. Current challenges and innovative developments in hydroxyapatite-based coatings on metallic materials for bone implantation: A review. Coatings 2020, 10, 1249. [CrossRef]

5. Chen, J.S. Characterization of sol-gel derived calcium hydroxyapatite coatings fabricated on patterned rough stainless steel surface. Coatings 2019, 9, 334.

6. O'Neill, R.; McCarthy, H.O.; Montufar, E.B.; Ginebra, M.P.; Wilson, D.I.; Lennon, A.; Dunne, N. Critical review: Injectability of calcium phosphate pastes and cements. Acta Biomater. 2017, 50, 1-19. [CrossRef] [PubMed]

7. Burguera, E.F.; Xu, H.H.; Sun, L. Injectable calcium phosphate cement: Effects of powder-to-liquid ratio and needle size. J. Biomed. Mater. Res. Part B Appl. Biomater. 2008, 84, 493-502. [CrossRef] [PubMed]

8. Montufar, E.B.; Maazouz, Y.; Ginebra, M.P. Relevance of the setting reaction to the injectability of tricalcium phosphate pastes. Acta Biomater. 2013, 9, 6188-6198. [CrossRef] [PubMed]

9. Luo, E.; Hu, J.; Bao, C.; Li, Y.; Tu, Q.; Murray, D.; Chen, J. Sustained release of adiponectin improves osteogenesis around hydroxyapatite implants by suppressing osteoclast activity in ovariectomized rabbits. Acta Biomater. 2012, 8, 734-743. [CrossRef] [PubMed]

10. Dorozhkin, S.V. Calcium orthophosphate cements for biomedical application. J. Mater. Sci. 2008, 43, 3028. [CrossRef]

11. Lakouraj, M.M.; Alipour, A.; Najafiroudbari, M.; Ojani, R. Fabrication of a nanocomposite based on pectin/polyaniline/graphene oxide as novel electrically conductive biomaterial for biosensing detection of hemoglobin. Mod. Approaches Mater. Sci. 2020, 2, 295-303.

12. Klein, R.; Tetzlaff, R.; Weiss, C.; Schäfer, M.K.; Tanner, M.; Wiedenhöfer, B.; Grafe, I.; Meeder, P.J.; Noeldge, G.; Nawroth, P.P. Osteointegration and resorption of intra- and extravertebral calcium phosphate cement. J. Spinal Disord. Tech. 2013, 1, E291-E296.

13. Sheikh, Z.; Abdallah, M.N.; Hanafi, A.A.; Misbahuddin, S.; Rashid, H.; Glogauer, M. Mechanisms of in vivo degradation and resorption of calcium phosphate based biomaterials. Materials 2015, 8, 7913-7925. [CrossRef] [PubMed]

14. Gumpert, R.; Bodo, K.; Spuller, E.; Poglitsch, T.; Bindl, R.; Ignatius, A.; Puchwein, P. Demineralization after balloon kyphoplasty with calcium phosphate cement: A histological evaluation in ten patients. Eur. Spine J. 2014, 23, 1361-1368. [CrossRef] [PubMed]

15. Maestretti, G.; Sutter, P.; Monnard, E.; Ciarpaglini, R.; Wahl, P.; Hoogewoud, H.; Gautier, E. A prospective study of percutaneous balloon kyphoplasty with calcium phosphate cement in traumatic vertebral fractures: 10-year results. Eur. Spine J. 2014, 23, 1354-1360. [CrossRef] [PubMed]

16. Vojtova, L.; Michlovska, L.; Valova, K.; Zboncak, M.; Trunec, M.; Castkova, K.; Krticka, M.; Pavlinakova, V.; Polacek, P.; Dzurov, M.; et al. The effect of the thermosensitive biodegradable PLGA-PEG-PLGA copolymer on the rheological, structural and mechanical properties of thixotropic self-hardening tricalcium phosphate cement. Int. J. Mol. Sci. 2019, 20, 391. [CrossRef] [PubMed]

17. Lanao, R.P.F.; Leeuwenburgh, S.C.; Wolke, J.G.; Jansen, J.A. In vitro degradation rate of apatitic calcium phosphate cement with incorporated PLGA microspheres. Acta Biomater. 2011, 7, 3459-3468. [CrossRef] [PubMed]

18. Lanao, R.P.F.; Sariibrahimoglu, K.; Wang, H.; Wolke, J.G.; Jansen, J.A.; Leeuwenburgh, S.C. Accelerated calcium phosphate cement degradation due to incorporation of glucono-delta-lactone microparticles. Tissue Eng. Part A 2014, 20, 378-388. [CrossRef] [PubMed] 
19. Albee, F.H. Studies in bone growth: Triple calcium phosphate as a stimulus to osteogenesis. Ann. Surg. 1920, 71, 32-39. [CrossRef] [PubMed]

20. Palmer, I.; Nelson, J.; Schatton, W.; Dunne, N.J.; Buchanan, F.J.; Clarke, S.A. Biocompatibility of calcium phosphate bone cement with optimized mechanical properties. J. Biomed. Mater. Res. Part B Appl. Biomater. 2016, 104, 308-315. [CrossRef] [PubMed] 